OPEN ACCESS

Edited by:

Weimin Mao,

Zhejiang Cancer Hospital, China

Reviewed by:

Alessandro Morabito,

Istituto Nazionale Tumori Fondazione

G. Pascale (IRCCS), Italy

Ke-Neng Chen,

Peking University Cancer Hospital,

China

Yang Yunhai,

Shanghai Jiaotong University, China

*Correspondence:

Da Chen

nuu2k@sina.com

Specialty section:

This article was submitted to

Thoracic Oncology,

a section of the journal

Frontiers in Oncology

Received: 21 February 2021

Accepted: 19 April 2021

Published: 04 May 2021

Citation:

Fang L, Zhao W, Ye B and Chen D (2021) Combination of Immune

Checkpoint Inhibitors and

Anti-Angiogenic Agents in Brain

Metastases From Non-Small

Cell Lung Cancer.

Front. Oncol. 11:670313.

doi: 10.3389/fonc.2021.670313

\section{Combination of Immune Checkpoint Inhibitors and Anti-Angiogenic Agents in Brain Metastases From Non-Small Cell Lung Cancer}

\author{
Likui Fang, Wuchen Zhao, Bo Ye and Da Chen* \\ Department of Thoracic Surgery, Affiliated Hangzhou Chest Hospital, Zhejiang University School of Medicine, \\ Hangzhou, China
}

Brain metastases remain a critical issue in the management of non-small cell lung cancer (NSCLC) because of the high frequency and poor prognosis, with survival rates often measured in just months. The local treatment approach remains the current standard of care, but management of multiple asymptomatic brain metastases always involves systemic therapy. Given that anti-angiogenic agents and immune checkpoint inhibitors (ICls) both target the tumor microenvironment (TME), this combination therapy has become a promising strategy in clinical practice. Increasing number of preclinical and clinical studies have shown remarkable anti-tumor activity of the combination therapy, but the efficacy in brain metastases is unclear due to the strict selection criteria adopted in most clinical trials. This review briefly summarizes the potential synergistic anti-tumor effect and clinical development of the combination of anti-angiogenic agents and ICls in NSCLC brain metastases, and discusses the existing challenges and problems.

Keywords: non-small cell lung cancer, brain metastases, immune checkpoint inhibitors, anti-angiogenesis, combination therapy

\section{INTRODUCTION}

Lung cancer is one of the most common malignant tumors and the leading cause of cancer-related mortality worldwide $(1,2)$. Non-small cell lung cancer (NSCLC) is the most frequent subtype of lung cancer and approximately 57\% patients with NSCLC are in advanced stage including 20\% presenting with brain metastases at the time of diagnosis (3). Brain metastases are also the common pattern of distant relapse after initial treatment $(4,5)$. Brain metastases are associated with poor prognosis and portend limited effective treatment options (6).

Current treatment strategies include local and systematic management. For the patients with symptomatic and immediately life-threatening brain metastases, surgical resection and radiotherapy are the major therapeutic approaches because of their relatively effective local control (7-9). However, surgery is typically reserved for intracranial hemorrhage, large lesions, and solitary brain metastases (10). Similarly, the use of stereotactic radiosurgery is limited by the number of metastatic lesions and is not suitable for the tumors which are larger than $4 \mathrm{~cm}$ or located in critical structures $(11,12)$. Whole brain radiotherapy is still the main method for the patients with multiple brain metastases or when stereotactic radiosurgery is not feasible (13). Although local 
treatment has an irreplaceable status in brain metastases currently, its toxic effects should warrant enough attention, such as cognitive decline and symptomatic radiation necrosis $(7,14,15)$. Moreover, local treatment could delay the initiation of systemic treatment, which would lead to the progression of primary tumors and compromise long-term outcomes.

Considering the limitations of local treatment, systematic therapy for NSCLC brain metastases has been explored due to its simultaneous treatment for both intracerebral and extracerebral diseases. Chemotherapy is not so often an effective approach for metastatic brain lesions, whereas tyrosine kinase inhibitors (TKIs) therapy such as Osimertinib in oncogene driven disease has shown a good activity also on brain metastases (16-18). In the era of immunotherapy, there is increasing evidence supporting the use of immune checkpoint inhibitors (ICIs) in the treatment of NSCLC brain metastases when no targetable driver mutation has been identified (19). Despite the encouraging data, only few patients respond to immunotherapy and additional combination treatment strategies are in urgent need. Given that both anti-angiogenesis and immune checkpoint blockade focus on targeting the tumor microenvironment (TME), the combination of ICIs and antiangiogenic agents has become an attractive strategy. This review summarizes the potential synergistic anti-tumor effect and clinical development of this combination therapy strategy in NSCLC brain metastases.

\section{POTENTIAL MECHANISMS}

Tumorigenesis involves a succession of genetic alterations which have been classified into eight distinctive and complementary biologic capabilities, including sustaining proliferative signaling, evading growth suppressors, deregulating cellular energetics, enabling replicative immortality, resisting cell death, inducing angiogenesis, avoiding immune destruction and activating invasion and metastasis (20). Therefore, angiogenesis and immune escape are two critical processes of tumorigenesis. Moreover, TME is widely accepted as an important regulator of cancer formation and progression. The tumor vasculature is a key component of the microenvironment that can be targeted through the use of anti-angiogenic agents. Blood vascular and lymphatic endothelial cells have important roles in regulating the microenvironment and modulating the immune response. Improving access to the tumor through vascular normalization with anti-angiogenic agents may prove an effective combination strategy with immunotherapy approaches, and this combination therapy could have synergistic effects on TME to inhibit tumorigenesis. However, even though TME is a potentially rich source of therapeutic targets, our knowledge of the brain TME lacks comprehensive and integrative analysis.

The brain has long been regarded as immune privileged organ because blood brain barrier (BBB) and blood cerebrospinal fluid barrier (BCB) limit the entry of immune cells from the periphery. However, the immune privileged status of brain has been recently challenged by the discovery of lymphatic vessels that connect the central nervous system (CNS) with the periphery and are able to carry both fluid and immune cells $(21,22)$. This discovery leads to a reassessment of long-held assumptions in neuroimmunology and sheds new light on the application of immunotherapy in brain metastases. Several in-depth studies of immune microenvironmental landscape within CNS have revealed disease-specific enrichment of immune cells, including tissue-resident microglia, infiltrating monocyte-derived macrophages, neutrophils, and T cells $(23,24)$. Principalcomponent analysis has confirmed that monocyte-derived macrophages, neutrophils, and CD4+ and CD8+ T cells are the major immune cell determinants of the TME landscape of lung cancer brain metastases (24). In addition, brain metastases can disrupt the integrity of the $\mathrm{BBB}$ and $\mathrm{BCB}$ and recruit different immune cells from the myeloid and lymphoid lineage to the CNS (25). Angiogenesis is one of the specific hallmarks of NSCLC brain metastases and pivotal for the progression of metastasizing lesions, which have been proven by the observations of human autopsy specimens $(26,27)$. In addition, the tumor vasculature has important immunomodulatory roles including preventing the immune rejection of tumors (28). There have been several clinical studies suggesting that inclusion of anti-angiogenic therapies should be evaluated in selected patients with asymptomatic NSCLC brain metastases (29, 30). These findings provide theoretical supports for the use of ICIs and anti-angiogenic agents in NSCLC brain metastases. The development of this combination strategy is based on the understandings of the interaction between these two therapeutic interventions and their effects on the TME.

\section{Anti-Angiogenic Agents Promote Anti-Tumor Immune Response}

Angiogenesis involves many signaling pathways, such as vascular endothelial growth factor (VEGF)-VEGF receptor (VEGFR), platelet derived growth factor (PDGF)-PDGF receptor (PDGFR) and fibroblast growth factor (FGF)-FGF receptor (FGFR). These signaling pathways influence multiple steps of the cancer immune response $(31,32)$ (Figure 1). VEGF is one of the most studied factors triggering angiogenesis. In the circulation, the level of VEGF was found to be inversely correlated with the level of mature dendritic cell (DC) which is the main antigen-presenting cell (33). VEGF-VEGFR signaling pathway could inhibit the transcriptional activation of nuclear factor- $\kappa \mathrm{B}$ to affect the differentiation and maturation of DCs (34, 35). Moreover, VEGF could also inhibit the antigen-presentation function of DCs by upregulating programmed cell death protein ligand-1 (PD-L1) expression on DCs (36). As a result, cancer antigens fail to be presented to $\mathrm{T}$ cells, leading to silence of cytotoxic T lymphocytes. In addition, PDGF could also restrain DC maturation (31). Anti-angiogenic agents could increase the level of mature DCs and enhance the uptake of antigen presentation, resulting in the promotion of anti-tumor immune response $(37,38)$.

$\mathrm{T}$ cell infiltration is widely accepted as a key component of adaptive cancer immune response. VEGF could inhibit the differentiation of CD8+ and CD4+ T cells from hematopoietic 


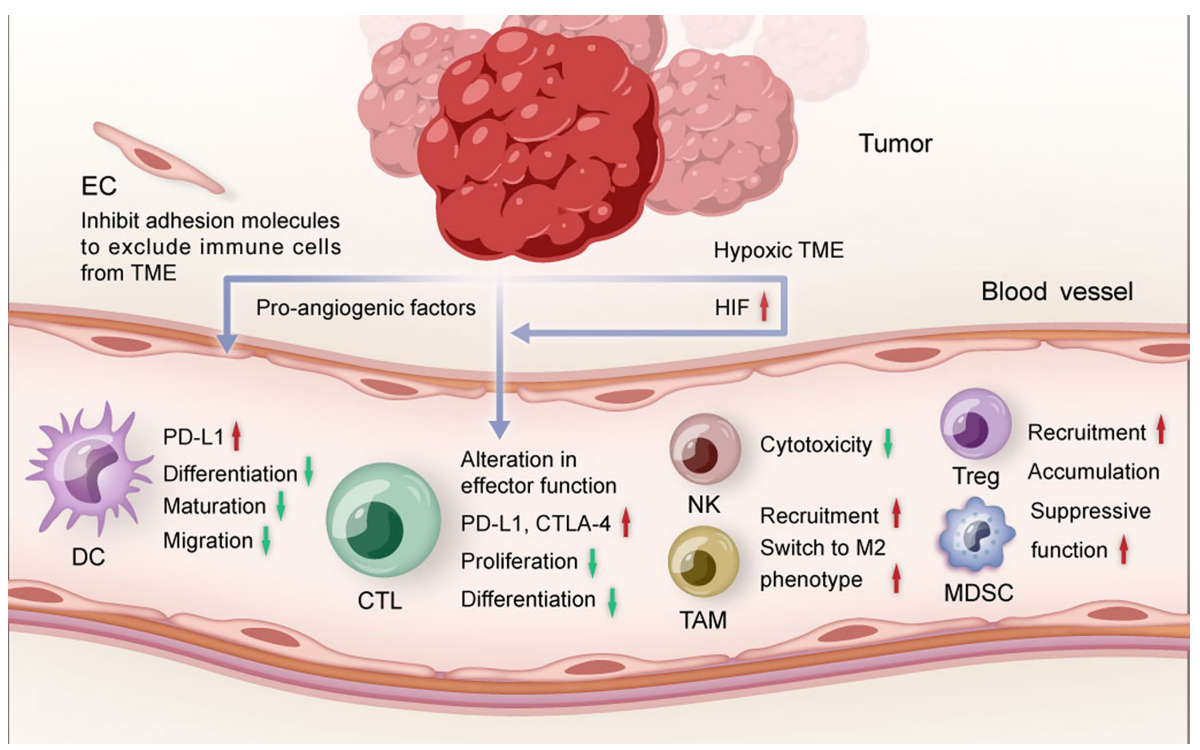

FIGURE 1 | The role of tumor angiogenesis in TME. Pro-angiogenic factors and hypoxia restrict the maturation and migration of dendritic cells, reduce the proliferation and differentiation of effector CTLs, and promote the recruitment of suppressive immune cells. TME, tumor microenvironment; DC, dendritic cell; CTL, cytotoxic T Iymphocytes; NK, natural killer cell; TAM, tumor-associated macrophage; Treg, regulatory T cell; MDSC, myeloid-derived suppressor cell; EC, endothelial cell; HIF, hypoxia-inducible factor.

progenitor cells and lead to the occurrence of T-cell deficiency (39). Moreover, activation of VEGF-VEGFR signaling pathway on CD8+T cells could induce $\mathrm{T}$ cell exhaustion and reduce $\mathrm{T}$ cell cytotoxicity by increasing the expression of programmed cell death protein 1 (PD-1) and cytotoxic T-lymphocyte-associated protein 4 (CTLA-4) (40). Similarly, natural killer cells (NK) cytotoxicity could be impaired by VEGF-VEGFR signaling pathway (41). Overexpressed VEGF could also inhibit the recruitment of type 1 helper $\mathrm{T}$ cells (Th) at tumor site but enhance the recruitment and proliferation of immunosuppressive cells including regulatory $\mathrm{T}$ cells (Treg) and myeloid-derived suppressor cells (MDSC) to promote the formation of immunosuppressive microenvironment (31).

The steps of immune cells infiltrating into the TME include entering the tumor vessels, attaching to the endothelial cells and finally migrate to the TME through the vascular wall (42). Angiogenic molecules are capable to regulate the expression of different adhesion molecules such as intercellular adhesion molecule-1 (ICAM-1) and vascular cell adhesion molecule 1 (VCAM1) to inhibit the transfer of immune cells to TME (28). Moreover, tumor endothelial cells could not only form a specific selective barrier to inhibit the penetration of certain immune cells (31), but also modulate the activity and variability of immune cells to regulate immunosuppression (32).

Abnormal tumor vasculature could aggravate the hypoxia in TME, leading to immune suppression through multiple mechanisms including recruitment of MDSCs, accumulation of Tregs (43) and activation of hypoxia-inducible factor (HIF) which is a critical factor of regulating angiogenesis and immune response (44). HIF could participate in innate and adaptive immunity. For example, HIF could promote recruitment of monocytes and M2 tumor-associated macrophages (TAMs) by upregulating the expression of nuclear factor- $\mathrm{\kappa B}$ (44). TAMs have emerged as prominent players in brain cancer (24). They are highly plastic cells that integrate input from cytokines, growth factors, and other stimuli, resulting in diverse activation states and cellular phenotypes, including promotion of invasion, angiogenesis, metastasis, and immune suppression (24). HIF could also inhibit DC maturation, inactivate cytotoxic $\mathrm{T}$ lymphocytes (CTLs) and target PD-L1 to evade anticancer immune responses (31).

Overall, the immunomodulatory effects of tumor vasculature are important targets in understanding and manipulating the TME. Anti-angiogenic therapy could not only normalize the tumor vasculature, but also transform the immunosuppressive TME to the immunosupportive one to improve anti-tumor immune response.

\section{ICls Enhance the Anti-Tumor Effects of Anti-Angiogenic Agents}

Tumor immune response is closely influenced by angiogenesis. Meanwhile, tumor angiogenesis also highly depends on immunosuppressive microenvironment. ICIs could activate immune cells to secret immune-mediating cytokine with antiangiogenesis effects to induce tumor vessel normalization (45). IFN- $\gamma$ is one of the important mediums during the process (Figure 2). For example, the activation of IFN- $\gamma$ signaling pathway on $\mathrm{CD} 8+\mathrm{T}$ cells might be one of the potential mechanisms of the vasculature-normalizing effect of ICIs (32). IFN- $\gamma$ could inhibit some pathways inducing angiogenesis, such as Notch signaling pathway, to effectively retard tumor growth (31). IFN- $\gamma$ could also reduce the VEGF secretion of tumor- 


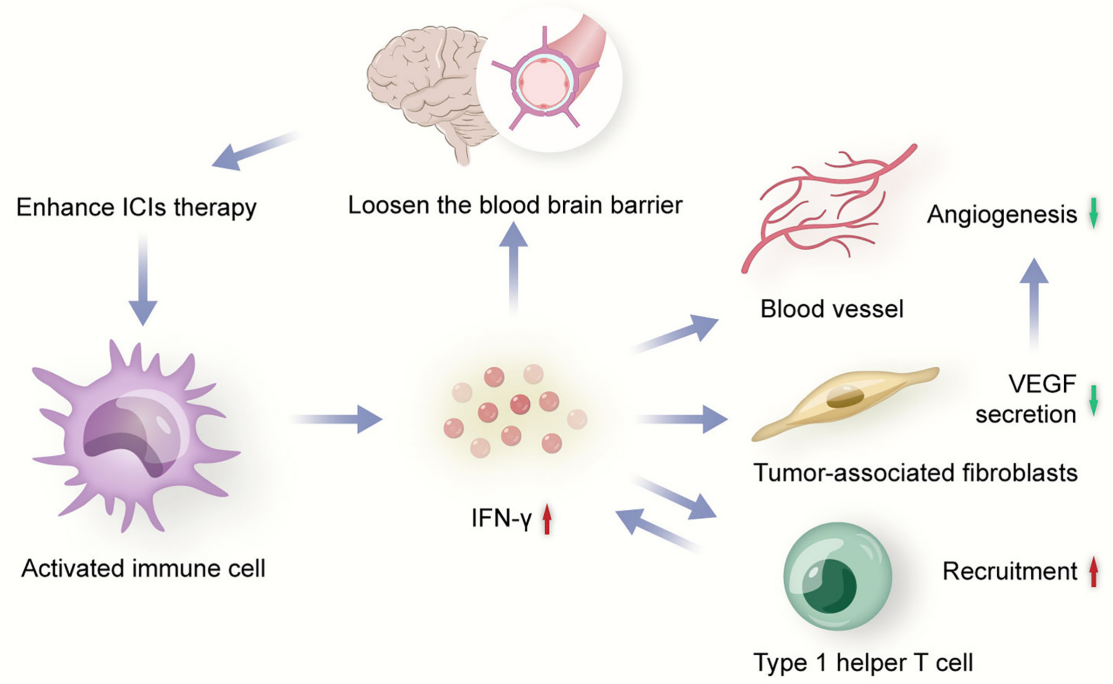

FIGURE 2 | Activated immune cell secrets IFN- $\gamma$ to inhibit angiogenesis. ICls, immune checkpoint inhibitors; VEGF, vascular endothelial growth factor.

associated fibroblasts to down-regulated angiogenesis (31). In addition, IFN- $\gamma$ could increase expression of CXCL9, CXCL10, and CXCL1 which recruit Th1 cells (46), and Th1 cells could secrete IFN- $\gamma$ in turn, which is significantly associated with vessel normalization (47). Besides, activated CD4+ T cells in the brain could loosen the $\mathrm{BBB}$ to circulating antibodies through local IFN- $\gamma$ production, which is a mechanism that anti-PD-1/PD-L1 therapy could potentially enhance (48).

Immunosuppressive cells could also stimulate tumor angiogenesis by cooperating with pro-angiogenic factors. For instance, MDSCs could enhance the proliferation and migration of endothelial cells by secreting VEGF, and promote tumor angiogenesis by inducing the production of matrix metalloproteinase 9 to act on the extracellular matrix (31). DC precursors could induce tumor angiogenesis in cooperation with VEGF-VEGFR signaling pathway which could further induce DC precursor endothelial-like specialization and migration to blood vessels (31). Moreover, through the expression and secretion of pro-angiogenic factors, some other myeloid cell subgroups might be also equipped with the ability to promote angiogenesis, including TAMs, Tregs, B cells, monocytes and neutrophils (31). These basic researches have provided the evidence that anti-angiogenic therapy could be more effective following the generation of an immunosupportive microenvironment.

Basic researches have suggested that immune response and angiogenesis are mutually regulated, and alleviated immunosuppression coupled with normalization of the tumor vasculature could achieve a loop of positive feedback that promotes each other (32). Some preclinical studies indicated that the efficacy of ICIs combined with anti-angiogenesis was significantly superior to monotherapy in advanced NSCLC. Sha Zhao et al. demonstrated that based on syngeneic lung cancer mouse model, low-dose apatinib could result in alleviating hypoxia, increasing infiltration of $\mathrm{CD} 8+\mathrm{T}$ cells, reducing recruitment of TAMs in tumor and decreasing TGF- $\beta$ level both in tumor and serum (49). They also found that combining low-dose apatinib with anti-PD-L1 antibody could significantly retard tumor growth and metastases, and induce prolonged survival in mouse models (49). Additionally, apatinib could improve the anti-tumor efficacy of anti-PD-1 therapy via upregulating PD-L1 expression in a syngeneic mouse model (50), which might provide a rationale for this combination strategy in the clinic.

\section{CLINICAL DATA}

Based on the synergistic effect on TME, the combination of ICIs and anti-angiogenic agents has been performed in advanced NSCLC. Although data are still immature, clinical benefits have been obtained from this combination strategy. The results of clinical trials investigating the combination effect of antiangiogenic agents and ICIs were presented in Table $\mathbf{1 .}$

Herbst et al. designed a multi-cohort phase I trial (NCT02443324) to assess the effect of ramucirumab plus pembrolizumab in the patients with advanced NSCLC with prior progression on systemic therapy (51). This trial enrolled 27 patients with $77.8 \%$ adenocarcinoma and $14.8 \%$ squamous cell carcinoma. Median progression-free survival (PFS) was 9.7 months and overall survival (OS) rate at 6 month was $84.9 \%$. Objective response rate (ORR) and disease control rate (DCR) were $30 \%$ and $85 \%$, respectively. Treatment related adverse events (TRAEs) occurred in $25(92.6 \%)$ patients with $18.5 \%$ grade 3 including adrenal insufficiency, delirium, hypertension, hyponatremia, infusion related reaction, proteinuria and respiratory failure. No grade 4-5 TRAEs occurred. In addition, Chu et al. conducted a phase Ib trial (NCT03628521) to evaluate 
TABLE 1 | Clinical trials investigating the combination effect of anti-angiogenic agents and ICls in NSCLC.

\begin{tabular}{|c|c|c|c|c|c|c|}
\hline Clinical trial & Phase & Histology & $\begin{array}{l}\text { Brain metas- } \\
\text { tases }\end{array}$ & Treatment & Results & TRAEs \\
\hline NCT02443324 & 1 & $\begin{array}{l}\text { Adenocarcinoma, } 21 / 27 \\
(77.8 \%) \\
\text { Squamous cell carcinoma, 4/27 } \\
(14.8 \%)\end{array}$ & NA & Pembrolizumab plus ramucirumab & $\begin{array}{l}\text { ORR, } 30 \% \\
\text { DCR, } 85 \% \\
\text { Median PFS, } \\
9.7 \mathrm{~m} \\
\text { OS rate at } 6 \mathrm{~m} \text {, } \\
84.9 \%\end{array}$ & $\begin{array}{l}\text { Total, 25/27 (92.6\%) } \\
\text { Grade 3, 5/27 } \\
\text { (18.5\%) } \\
\text { Grade 4-5, 0/27 } \\
(0 \%)\end{array}$ \\
\hline NCT03628521 & $\mathrm{lb}$ & $\begin{array}{l}\text { Squamous cell carcinoma, 12/ } \\
22(54.5 \%) \\
\text { Adenocarcinoma, 9/22 (40.9\%) }\end{array}$ & 4/22 (18.2\%) & Sintilimab plus anlotinib & $\begin{array}{l}\text { ORR, } 72.7 \% \\
\text { DCR, } 100 \% \\
\text { Median PFS, } \\
15 \mathrm{~m}\end{array}$ & $\begin{array}{l}\text { Total, } 22 / 22 \text { (100\%) } \\
\text { Grade 3, 12/22 } \\
\text { (54.5\%) } \\
\text { Grade } 4-5,1 / 22 \\
(4.5 \%)\end{array}$ \\
\hline NCT01454102 & I & Non-squamous cell carcinoma & NA & Nivolumab plus bevacizumab & $\begin{array}{l}\text { ORR, } 8 \% \\
\text { DCR, } 58 \% \\
\text { Median PFS, } \\
37.1 \mathrm{w} \\
\text { OS rate at } 1 \mathrm{y}, \\
75 \%\end{array}$ & $\begin{array}{l}\text { Total, 11/12 (91.7\%) } \\
\text { Grade 3, 4/12 } \\
\text { (33.3\%) } \\
\text { Grade 4-5, 0/12 } \\
\text { (0\%) }\end{array}$ \\
\hline IMpower150 & III & Non-squamous cell carcinoma & NA & $\begin{array}{l}\text { Atezolizumab plus bevacizumab plus carboplatin } \\
\text { plus paclitaxel }\end{array}$ & $\begin{array}{l}\text { ORR, 63.5\% } \\
\text { DCR, 85.3\% } \\
\text { Median PFS, } \\
8.3 \mathrm{~m} \\
\text { Median OS, } \\
19.2 \mathrm{~m}\end{array}$ & $\begin{array}{l}\text { Total, 371/393 } \\
(94.4 \%) \\
\text { Grade 3-4, 219/393 } \\
(55.7 \%) \\
\text { Grade 5, 11/393 } \\
(2.8 \%)\end{array}$ \\
\hline NCT02039674 & I & Non-squamous cell carcinoma & $4 / 25(16 \%)$ & $\begin{array}{l}\text { Pembrolizumab plus bevacizumab plus } \\
\text { carboplatin plus paclitaxel }\end{array}$ & $\begin{array}{l}\text { ORR, 56\% } \\
\text { DCR, 76\% } \\
\text { Median PFS, } \\
7.1 \mathrm{~m} \\
\text { Median OS, } \\
16.7 \mathrm{~m}\end{array}$ & $\begin{array}{l}\text { Total, 23/24 (95.8\%) } \\
\text { Grade 3-4, 10/24 } \\
(41.7 \%) \\
\text { Grade 5, 0/24 (0\%) }\end{array}$ \\
\hline
\end{tabular}

NSCLC, non-small cell lung cancer; $m$, month(s); w, weeks; $y$, year; NA, not applicable; ICls, immune checkpoint inhibitors; ORR, objective response rate; DCR, disease control rate; PFS, progression-free survival; OS, overall survival; TRAEs, treatment related adverse events.

chemo-free first-line strategy of sintilimab combining anlotinib in treatment-naive and stage IIIB/IV NSCLC patients (52). Twenty-two patients were enrolled in the study and four had baseline brain metastases. The results showed high ORR (72.7\%) and DCR (100\%) with acceptable tolerability. The incidence rate of grade 3 TRAEs was $54.5 \%$. No grade 4 TRAEs were observed, and one case of grade 5 immune-related pneumonitis occurred. The most common TRAEs were hemorrhage $(59.1 \%)$, hypothyroidism (50.0\%) and hyperuricemia (40.9\%). Moreover, Rizvi et al. reported preliminary results from a phase I study (NCT01454102) evaluating the efficacy and safety of nivolumab plus bevacizumab as maintenance therapy in advanced NSCLC without progress on first-line platinum based chemotherapy (53). Median PFS was 37.1 weeks and 1 -year OS rate was $75 \%$. TRAEs occurred in 11/12 (91.7\%) patients with $33.3 \%$ grade 3 and no grade 4 TRAEs. Grade 3 adverse events included pneumonitis, cough and tubulointerstitial nephritis.

IMpower150, a phase III randomized trial, showed a significant prognostic improvement with the addition of atezolizumab to bevacizumab and chemotherapy as first-line treatment for nonsquamous metastatic NSCLC (54). This clinical study enrolled a total of 1202 patients and randomly assigned them to three group, atezolizumab plus bevacizumab plus carboplatin plus paclitaxel (ABCP group, 400 patients), atezolizumab plus carboplatin plus paclitaxel (ACP group,
402 patients) and bevacizumab plus carboplatin plus paclitaxel (BCP group, 400 patients). The results indicated that ABCP group had higher rate of PFS at 12 months and objective response than $\mathrm{BCP}$ group, regardless of the $\mathrm{PD}-\mathrm{L} 1$ expression status. It was worth mentioning that $\mathrm{ABCP}$ group also showed significant survival benefit in comparison to $\mathrm{BCP}$ group in the patients with sensitizing EGFR mutations and liver metastases (55). However, the frequency of TRAEs did not increase with the addition of atezolizumab and the safety profile was consistent with previously reported safety risks of the individual medicines (54). Although IMpower150 study confirmed successful combination of ICIs and anti-angiogenic agents in metastatic NSCLC, this study excluded patients if they had untreated metastases of the central nervous system. In contrast, a multicohort phase I study (NCT02039674) explored the anti-tumor activity and safety of pembrolizumab plus carboplatin-paclitaxelbevacizumab in advanced non-squamous NSCLC without prior systemic therapy (56). This study randomly assigned patients into 3 cohorts (A, B and $C$ ) and the patients in cohort $B$ received pembrolizumab plus carboplatin-paclitaxel-bevacizumab. Cohort B enrolled 25 patients with 4 (16\%) brain metastases. ORR was 56\% with 1 (4\%) complete response and 13 (52\%) partial response. Median PFS was 7.1 months and median OS was 16.7 months. TRAEs occurred in $95.8 \%$ patients and most events were of mild-to-moderate severity. It should be noted that TRAEs resulted in discontinuation of study treatment in 5 cases 
in cohort B, including neutropenia, autoimmune colitis, diarrhea, drug hypersensitivity, and pneumonitis.

A real-world retrospective study enrolled 69 patients with NSCLC to explore the efficacy of ICIs combining antiangiogenesis therapy (57). Sixty-three (91.3\%) patients were at stage IV and 16 (23.2\%) had sensitizing EGFR mutations. Twenty-nine (42\%) patients received nivolumab and $40(58 \%)$ received pembrolizumab. Bevacizumab was used in 45 (65.2\%) patients and the remaining patients received apatinib, anlotinib or endostar. ORR was $31.9 \%$ and DCR was $89.9 \%$. Median PFS was 8.37 months, while median OS was not reached. It should be noted that the patients receiving combined therapy within 6 months after diagnosis had better ORR than those exceeding 6 months $(59.1 \%$ vs. $19.1 \%, \mathrm{P}=0.001)$. These results suggested that it would be better to apply ICIs plus anti-angiogenic agents at the early stage after initial diagnosis. TRAEs appeared in $62 \%$ of patients. Most TRAEs were grade 1-2 with only 2 (2.9\%) grade 3 (pneumonitis, diarrhea) and no grade 4-5 events. The most common adverse events were fatigue, decreased appetite and nausea.

The combination of ICIs and anti-angiogenic agents showed encouraging anti-tumor activity and tolerable safety profile. Due to the potential neurological sequelae, patients with brain metastases were often excluded from clinical trials. Major ongoing or planned trials investigating ICIs in combination with anti-angiogenic agents in patients with NSCLC (Table 2) include NCT03377023 (a trial of nivolumab plus ipilimumab plus nintedanib), NCT03689855 (a trial of atezolizumab plus ramucirumab), NCT03527108 (a trial of nivolumab plus ramucirumab) and NCT02681549 (a trial of pembrolizumab plus bevacizumab) (58). However, at the time of writing, there are no published trial data from prospective randomized controlled trials focusing on the effects of this combination strategy in NSCLC patients with brain metastases which warrant further studies.

\section{PREDICTIVE INDICATORS}

Despite the promising prospect of immunotherapy and antiangiogenesis therapy in NSCLC brain metastases, this combination strategy still faces many challenges, one of which is identifying ideal predictive indicators to screen suitable populations. As for anti-angiogenesis therapy, circulating VEGF-A level was evaluated for the prognostic and predictive value in a retrospective analysis (59). This study included five trials involving three types of cancer, AVF2107 (colorectal cancer), E4599 (NSCLC), AVAiL (NSCLC), AVOREN (renal cell carcinoma) and AVF2938 (renal cell carcinoma). In E4599 trial, bevacizumab-based treatment was predictive for PFS benefit in high circulating VEGF-A group $(>36 \mathrm{pg} / \mathrm{mL})$ but not in low VEGF-A group. By contrast, circulating VEGF-A level (cutoff value, $45 \mathrm{pg} / \mathrm{mL}$ ) was not prognostic for PFS and OS in AVAiL trial. Other biomarkers such as VEGFR-2, FGF-2 and IL8 were proposed and investigated, but none could predict response to anti-angiogenesis therapy (60). Several studies indicated that anti-angiogenic TRAEs and the number of circulating endothelial cells were positively associated with the clinical benefit $(60,61)$, but none were validated for routine clinical use. Similarly, the use of ICIs for the treatment of intracranial metastatic tumors also requires effective predictive indicators. Previous studies have proven that the expression of PD-L1 and the presence of tumor-infiltrating lymphocytes (TILs) within TME are considered prognostic and predictive markers in patients treated with immunotherapy $(62,63)$. However, the PD-L1 expression and the presence of TILs might be different in CNS when compared with extracranial sites, with lower PD-L1 expression and less TILs infiltration in brain metastases compared with matched NSCLC primary tumors (64). Tumor mutational burden (TMB) was also a useful biomarker for response to ICIs in advanced NSCLC (65), but its value in brain metastases remains unclear. DNA mismatch repair-deficient (dMMR)/microsatellite instabilityhigh (MSI-H) has also been reported to be able to predict the efficacy of ICIs, but the low frequency in NSCLC limits its clinical application (66).

As for the combination therapy, the phase Ib trial (NCT03628521) indicated that the patients with TMB $\geq 10$ mutations per megabase showed higher ORR than those with $\mathrm{TMB}<10$ mutations per megabase $(85.7 \%$ vs. 63.6\%) (52). It is worth mentioning that ORR in the patients with positive and negative PD-L1 expression was $69.2 \%$ and $75 \%$, respectively (67). The phase I trial (NCT02039674) showed that patients with PDL1 TPS $\geq 50 \%$ were seemed to have higher ORR than those with PD-L1 TPS <50\% (75\% vs. 47\%) (56). IMpower 150 also suggested that high expression of an effector T-cell (Teff) gene signature in the tumor was associated with survival benefit (54). However, in comparison to primary tumor of NSCLC, brain metastasis lesions displayed significant downregulation of genes related to immune response and immune cell activation (68). In addition, it is unclear whether aspects of the tumor vasculature are different in tumors that respond to immunotherapy and

TABLE 2 | Major ongoing or planned trials investigating ICls in combination with anti-angiogenic agents in patients with NSCLC.

\begin{tabular}{|c|c|c|c|c|}
\hline Clinical trial & Phase & Treatment (arm of combination therapy) & Planned patients & Primary objective \\
\hline NCT03377023 & $|/| \mid$ & Nivolumab plus ipilimumab plus nintedanib & Advanced or metastatic NSCLC & MTD, ORR \\
\hline NCT03689855 & $\|$ & Atezolizumab plus ramucirumab & Squamous or non-squamous NSCLC & ORR \\
\hline NCT03527108 & $\|$ & Nivolumab plus ramucirumab & Refractory or recurrent advanced NSCLC & DCR \\
\hline NCT02681549 & $\|$ & Pembrolizumab plus bevacizumab & Metastatic melanoma or NSCLC & BMRR \\
\hline
\end{tabular}

ICls, immune checkpoint inhibitors; NSCLC, non-small cell lung cancer; MTD, maximum tolerated dose; ORR, objective response rate; DCR, disease control rate; BMRR, brain metastasis response rate. 
those that do not, and if features such as hypoxia or production of pro-angiogenic factors may serve as predictive biomarkers. These problems suggest that it is unlikely to precisely predict the efficacy of immunotherapy and anti-angiogenesis combination in brain metastases through current biomarkers. The specific predictive indicators to distinguish appropriate population need further exploration.

\section{DISCUSSION}

Given recent advances in immunotherapy, emerging clinical evidence suggests that ICIs have anti-tumor effects in brain metastases from NSCLC. The OAK study showed that the hazard ratio (HR) for OS with atezolizumab vs. docetaxel was 0.73 for the overall population, 0.74 for patients without brain metastases, and 0.54 for patients with brain metastases (69). Similarly, the KEYNOTE-189 study comparing pembrolizumab plus chemotherapy with chemotherapy alone indicated that the HR for OS was 0.36 for patients with brain metastases, with 0.49 for the overall population and 0.53 for patients without brain metastases (70). A pooled analysis of CheckMate 063, 017 and 057 also demonstrated that nivolumab showed a survival advantage in second-line therapy for stable brain metastases when compared with docetaxel (71). Beyond oncogene-driven NSCLC, ICIs have recently shown promising activity in the CNS in patients with NSCLC brain metastases.

Despite the significant benefits of immunotherapy, there are still some problems such as limited patient response rates and drug resistance. Because of both targeting aspects of the TME, immunotherapy and anti-angiogenesis are expected to mutually enhance the anti-tumor effect through reprogramming the TME from immunosuppressive to immunosupportive, but whether this combination can improve response rate or delay drug resistance of monotherapy remains unclear and needs further clinical studies. Tumors can be categorized as inflamed and noninflamed phenotypes based on the spatial localization of immune cells with respect to the tumor and stromal compartments (72). Almost all relevant therapeutic advances in the field of immunotherapy have been achieved in inflamed tumors, while non-inflamed tumors tend to respond poorly to ICIs (72). Whether anti-angiogenic therapy could expand the benefits of immune checkpoint inhibition to non-inflamed tumors requires additional researches. Clinically, steroids are frequently used in NSCLC patients with brain metastases with the aim of palliating cancer-related symptoms, but the use of steroids is associated with a lower efficacy of ICIs and a worse outcome (73). A retrospective study suggested that anlotinib could potentially replace glucocorticoids and effectively improve edema from brain metastases but this study only included 13 NSCLC

\section{REFERENCES}

1. Siegel RL, Miller KD, Jemal A. Cancer Statistics, 2020. CA Cancer J Clin (2020) 70(1):7-30. doi: 10.3322/caac.21590 patients with 23 brain metastases (74). Whether antiangiogenesis can indeed counteract the negative effect of steroids needs further research. Hyperprogression is defined as rapid disease progression during immunotherapy, which is associated with poor survival outcome (75). In theory, rapidly proliferative cancer cells need an abundance of blood supply for nutrition, while bevacizumab could starve these cells of blood supply and nutrients and provide potential benefit (76). However, the clinical data is absent and needs further study.

Although the preliminary clinical results have suggested that immunotherapy and anti-angiogenesis combination could potentially provide significant activity against brain metastases, the field of this combination strategy faces many challenges in the pursuit of overcoming the defect of monotherapy and improving the outcome of patients. Firstly, there are various combination regimens involving ICIs (PD-1, PD-L1 and CTLA4 inhibitors) and anti-angiogenic agents (anti-VEGF antibody, anti-VEGFR antibody and VEGFR TKIs). Which combination regimen is most effective for brain metastases remains to be answered by more data. Secondly, early phase clinical studies have reported the use time and the dosage of ICIs and antiangiogenic agents (51-53), and the use of anti-angiogenic agents prior ICIs was seemed to be more beneficial in vitro and vivo experiments (77). However, no studies have analyzed the changes of pharmacokinetic and pharmacodynamic profiles of each agent after combinational use. The optimal time and sequence of each agent in the combination are currently unknown. The appropriate dosage of each agent also remains unclear. Thirdly, although preliminary studies have showed acceptable toxicities and tolerance of the combination therapy, those studies are at early phase and the samples are small. The toxicities still require close attention. Finally, lacking of efficient and sensitive predictive indicators for the combination therapy leads to difficult selection of optimal candidates.

In conclusion, although resolving the above problems requires a long distance, the combination of ICIs and antiangiogenic agents has opened a new door for the treatment of NSCLC patients with brain metastases, and is expected to change the clinical management of those patients in the near future. Further studies are urgently needed to obtain the definitive data for the use of this combination strategy in clinic and facilitate the development of the optimal combination algorithm.

\section{AUTHOR CONTRIBUTIONS}

LF and DC performed a literature search, interpreted data, and wrote the manuscript. WZ and BY supervised and contributed to the writing process. All authors contributed to the article and approved the submitted version.

2. Bray F, Ferlay J, Soerjomataram I, Siegel RL, Torre LA, Jemal A. Globa Cancer Statistics 2018: GLOBOCAN Estimates of Incidence and Mortality Worldwide for 36 Cancers in 185 Countries. CA Cancer J Clin (2018) 68 (6):394-424. doi: 10.3322/caac.21492 
3. Davis FG, Dolecek TA, McCarthy BJ, Villano JL. Toward Determining the Lifetime Occurrence of Metastatic Brain Tumors Estimated From 2007 United States Cancer Incidence Data. Neuro-oncology (2012) 14(9):1171-7. doi: 10.1093/neuonc/nos152

4. Churilla TM, Weiss SE. Emerging Trends in the Management of Brain Metastases From Non-small Cell Lung Cancer. Curr Oncol Rep (2018) 20 (7):54. doi: 10.1007/s11912-018-0695-9

5. Chen AM, Jahan TM, Jablons DM, Garcia J, Larson DA. Risk of Cerebral Metastases and Neurological Death After Pathological Complete Response to Neoadjuvant Therapy for Locally Advanced Nonsmall-Cell Lung Cancer: Clinical Implications for the Subsequent Management of the Brain. Cancer (2007) 109(8):1668-75. doi: 10.1002/cncr.22565

6. Sperduto PW, Kased N, Roberge D, Xu Z, Shanley R, Luo X, et al. Summary Report on the Graded Prognostic Assessment: An Accurate and Facile Diagnosis-Specific Tool to Estimate Survival for Patients With Brain Metastases. J Clin Oncol (2012) 30(4):419-25. doi: 10.1200/JCO.2011.38.0527

7. Alomari A, Rauch PJ, Orsaria M, Minja FJ, Chiang VL, Vortmeyer AO. Radiologic and Histologic Consequences of Radiosurgery for Brain Tumors. J Neuro-oncol (2014) 117(1):33-42. doi: 10.1007/s11060-014-1359-8

8. Redmond AJ, Diluna ML, Hebert R, Moliterno JA, Desai R, Knisely JP, et al. Gamma Knife Surgery for the Treatment of Melanoma Metastases: The Effect of Intratumoral Hemorrhage on Survival. J Neurosurg (2008) 109 Suppl:99105. doi: $10.3171 /$ JNS/2008/109/12/S16

9. Bansal P, Rusthoven C, Boumber Y, Gan GN. The Role of Local Ablative Therapy in Oligometastatic non-Small-Cell Lung Cancer: Hype or Hope. Future Oncol (Lond Engl) (2016) 12(23):2713-27. doi: 10.2217/fon-2016-0219

10. Flanigan JC, Jilaveanu LB, Chiang VL, Kluger HM. Advances in Therapy for Melanoma Brain Metastases. Clin Dermatol (2013) 31(3):264-81. doi: 10.1016/j.clindermatol.2012.08.008

11. Otake S, Goto T. Stereotactic Radiotherapy for Oligometastasis. Cancers (2019) 11(2):133-45. doi: 10.3390/cancers11020133

12. Collen C, Christian N, Schallier D, Meysman M, Duchateau M, Storme G, et al. Phase II Study of Stereotactic Body Radiotherapy to Primary Tumor and Metastatic Locations in Oligometastatic Nonsmall-Cell Lung Cancer Patients. Ann Oncol (2014) 25(10):1954-9. doi: 10.1093/annonc/mdu370

13. Li W, Yu H. Separating or Combining Immune Checkpoint Inhibitors (Icis) and Radiotherapy in the Treatment of NSCLC Brain Metastases. J Cancer Res Clin Oncol (2020) 146(1):137-52. doi: 10.1007/s00432-019-03094-9

14. Greene-Schloesser D, Robbins ME. Radiation-Induced Cognitive Impairment-From Bench to Bedside. Neuro-oncology (2012) 14(Suppl 4): iv37-44. doi: 10.1093/neuonc/nos196

15. Khan AJ, Dicker AP. On the Merits and Limitations of Whole-Brain Radiation Therapy. J Clin Oncol (2013) 31(1):11-3. doi: 10.1200/JCO.2012.46.0410

16. Reungwetwattana T, Nakagawa K, Cho BC, Cobo M, Cho EK, Bertolini A, et al. Cns Response to Osimertinib Versus Standard Epidermal Growth Factor Receptor Tyrosine Kinase Inhibitors in Patients With Untreated Egfr-Mutated Advanced non-Small-Cell Lung Cancer. J Clin Oncol (2018) 36(33):3290. doi: 10.1200/JCO.2018.78.3118

17. Gadgeel S, Peters S, Mok T, Shaw AT, Kim DW, Ou SI, et al. Alectinib Versus Crizotinib in Treatment-Naive Anaplastic Lymphoma Kinase-Positive (ALK +) non-Small-Cell Lung Cancer: CNS Efficacy Results From the ALEX Study. Ann Oncol (2018) 29(11):2214-22. doi: 10.1093/annonc/mdy405

18. Ernani V, Stinchcombe TE. Management of Brain Metastases in Non-Small-Cell Lung Cancer. J Oncol Pract (2019) 15(11):563-70. doi: 10.1200/JOP.19.00357

19. Wang S, Hu C, Xie F, Liu Y. Use of Programmed Death Receptor-1 and/or Programmed Death Ligand 1 Inhibitors for the Treatment of Brain Metastasis of Lung Cancer. OncoTargets Ther (2020) 13:667-83. doi: 10.2147/OTT.S235714

20. Hanahan D, Weinberg RA. Hallmarks of Cancer: The Next Generation. Cell (2011) 144(5):646-74. doi: 10.1016/j.cell.2011.02.013

21. Louveau A, Smirnov I, Keyes TJ, Eccles JD, Rouhani SJ, Peske JD, et al. Structural and Functional Features of Central Nervous System Lymphatic Vessels. Nature (2015) 523(7560):337-41. doi: 10.1038/nature14432

22. Raper D, Louveau A, Kipnis J. How Do Meningeal Lymphatic Vessels Drain the CNS? Trends Neurosci (2016) 39(9):581-6. doi: 10.1016/j.tins.2016.07.001

23. Quail DF, Joyce JA. The Microenvironmental Landscape of Brain Tumors. Cancer Cell (2017) 31(3):326-41. doi: 10.1016/j.ccell.2017.02.009

24. Klemm F, Maas RR, Bowman RL, Kornete M, Soukup K, Nassiri S, et al. Interrogation of the Microenvironmental Landscape in Brain Tumors Reveals
Disease-Specific Alterations of Immune Cells. Cell (2020) 181(7):1643-60 e17. doi: $10.1016 /$ j.cell.2020.05.007

25. Schulz M, Salamero-Boix A, Niesel K, Alekseeva T, Sevenich L. Microenvironmental Regulation of Tumor Progression and Therapeutic Response in Brain Metastasis. Front Immunol (2019) 10:1713. doi: 10.3389/fimmu.2019.01713

26. Berghoff AS, Rajky O, Winkler F, Bartsch R, Furtner J, Hainfellner JA, et al. Invasion Patterns in Brain Metastases of Solid Cancers. Neuro-oncology (2013) 15(12):1664-72. doi: 10.1093/neuonc/not112

27. Berghoff AS, Ilhan-Mutlu A, Dinhof C, Magerle M, Hackl M, Widhalm G, et al. Differential Role of Angiogenesis and Tumour Cell Proliferation in Brain Metastases According to Primary Tumour Type: Analysis of 639 Cases. Neuropathol Appl Neurobiol (2015) 41(2):e41-55. doi: 10.1111/nan.12185

28. Hendry SA, Farnsworth RH, Solomon B, Achen MG, Stacker SA, Fox SB. The Role of the Tumor Vasculature in the Host Immune Response: Implications for Therapeutic Strategies Targeting the Tumor Microenvironment. Front Immunol (2016) 7:621. doi: 10.3389/fimmu.2016.00621

29. Berghoff AS, Preusser M. Anti-Angiogenic Therapies in Brain Metastases. Memo (2018) 11(1):14-7. doi: 10.1007/s12254-018-0384-2

30. Shukla NA, Yan MN, Hanna N. The Story of Angiogenesis Inhibitors in Nonsmall-cell Lung Cancer: The Past, Present, and Future. Clin Lung Cancer (2020) 21(4):308-13. doi: 10.1016/j.cllc.2020.02.024

31. Guo F, Cui J. Anti-Angiogenesis: Opening a New Window for Immunotherapy. Life Sci (2020) 258:118163. doi: 10.1016/j.lfs.2020.118163

32. Song Y, Fu Y, Xie Q, Zhu B, Wang J, Zhang B. Anti-Angiogenic Agents in Combination With Immune Checkpoint Inhibitors: A Promising Strategy for Cancer Treatment. Front Immunol (2020) 11:1956. doi: 10.3389/fimmu.2020.01956

33. Galati D, Zanotta S, Canora A, Polistina GE, Nicoletta C, Ghinassi G, et al. Severe Depletion of Peripheral Blood Dendritic Cell Subsets in Obstructive Sleep Apnea Patients: A New Link With Cancer? Cytokine (2020) 125:154831. doi: 10.1016/j.cyto.2019.154831

34. Aggarwal V, Tuli HS, Varol A, Thakral F, Yerer MB, Sak K, et al. Role of Reactive Oxygen Species in Cancer Progression: Molecular Mechanisms and Recent Advancements. Biomolecules (2019) 9(11):735-60. doi: 10.3390/ biom9110735

35. Alfaro C, Suarez N, Gonzalez A, Solano S, Erro L, Dubrot J, et al. Influence of Bevacizumab, Sunitinib and Sorafenib as Single Agents or in Combination on the Inhibitory Effects of VEGF on Human Dendritic Cell Differentiation From Monocytes. Br J Cancer (2009) 100(7):1111-9. doi: 10.1038/sj.bjc.6604965

36. Curiel TJ, Wei S, Dong H, Alvarez X, Cheng P, Mottram P, et al. Blockade of B7-H1 Improves Myeloid Dendritic Cell-Mediated Antitumor Immunity. Nat Med (2003) 9(5):562-7. doi: 10.1038/nm863

37. Long J, Hu Z, Xue H, Wang Y, Chen J, Tang F, et al. Vascular Endothelial Growth Factor (VEGF) Impairs the Motility and Immune Function of Human Mature Dendritic Cells Through the VEGF Receptor 2-RhoA-cofilin1 Pathway. Cancer Sci (2019) 110(8):2357-67. doi: 10.1111/cas.14091

38. Yang J, Yan J, Liu B. Targeting VEGF/VEGFR to Modulate Antitumor Immunity. Front Immunol (2018) 9:978. doi: 10.3389/fimmu.2018.00978

39. Ohm JE, Gabrilovich DI, Sempowski GD, Kisseleva E, Parman KS, Nadaf S, et al. VEGF Inhibits T-cell Development and may Contribute to TumorInduced Immune Suppression. Blood (2003) 101(12):4878-86. doi: 10.1182/ blood-2002-07-1956

40. Voron T, Colussi O, Marcheteau E, Pernot S, Nizard M, Pointet AL, et al. VEGF-a Modulates Expression of Inhibitory Checkpoints on CD8+ T Cells in Tumors. J Exp Med (2015) 212(2):139-48. doi: 10.1084/jem.20140559

41. Lee JY, Park S, Min WS, Kim HJ. Restoration of Natural Killer Cell Cytotoxicity by VEGFR-3 Inhibition in Myelogenous Leukemia. Cancer Lett (2014) 354(2):281-9. doi: 10.1016/j.canlet.2014.08.027

42. Chen DS, Mellman I. Oncology Meets Immunology: The Cancer-Immunity Cycle. Immunity (2013) 39(1):1-10. doi: 10.1016/j.immuni.2013.07.012

43. Facciabene A, Peng X, Hagemann IS, Balint K, Barchetti A, Wang LP, et al. Tumour Hypoxia Promotes Tolerance and Angiogenesis Via CCL28 and T (reg) Cells. Nature (2011) 475(7355):226-30. doi: 10.1038/nature10169

44. Palazon A, Goldrath AW, Nizet V, Johnson RS. HIF Transcription Factors, Inflammation, and Immunity. Immunity (2014) 41(4):518-28. doi: 10.1016/ j.immuni.2014.09.008

45. Yi M, Jiao D, Qin S, Chu Q, Wu K, Li A. Synergistic Effect of Immune Checkpoint Blockade and Anti-Angiogenesis in Cancer Treatment. Mol Cancer (2019) 18(1):60. doi: 10.1186/s12943-019-0974-6 
46. Zheng X, Fang Z, Liu X, Deng S, Zhou P, Wang X, et al. Increased Vessel Perfusion Predicts the Efficacy of Immune Checkpoint Blockade. J Clin Invest (2018) 128(5):2104-15. doi: 10.1172/JCI96582

47. Tian L, Goldstein A, Wang H, Ching Lo H, Sun Kim I, Welte T, et al. Mutual Regulation of Tumour Vessel Normalization and Immunostimulatory Reprogramming. Nature (2017) 544(7649):250-4. doi: 10.1038/nature21724

48. Iwasaki A. Immune Regulation of Antibody Access to Neuronal Tissues. Trends Mol Med (2017) 23(3):227-45. doi: 10.1016/j.molmed.2017.01.004

49. Zhao S, Ren S, Jiang T, Zhu B, Li X, Zhao C, et al. Low-Dose Apatinib Optimizes Tumor Microenvironment and Potentiates Antitumor Effect of PD-1/PD-L1 Blockade in Lung Cancer. Cancer Immunol Res (2019) 7(4):63043. doi: 10.1158/2326-6066.CIR-17-0640

50. Cai X, Wei B, Li L, Chen X, Liu W, Cui J, et al. Apatinib Enhanced anti-PD-1 Therapy for Colon Cancer in Mice Via Promoting PD-L1 Expression. Int Immunopharmacol (2020) 88:106858. doi: 10.1016/j.intimp.2020.106858

51. Herbst RS, Martin-Liberal J, Calvo E, Isambert N, Bendell J, Cassier P, et al. Previously Treated Advanced NSCLC Cohort From a Multi-Disease Phase 1 Study of Ramucirumab (R) Plus Pembrolizumab (P): Efficacy and Safety Data. Ann Oncol (2017) 28:ii32-ii3. doi: 10.1093/annonc/mdx091.010

52. Chu T, Zhong R, Zhong H, Zhang B, Zhang W, Shi C, et al. Phase Ib Study of Sintilimab Plus Anlotinib as First-line Therapy in Patients With Advanced non-Small Cell Lung Cancer. J Thorac Oncol (2020) 16(4):643-52. doi: 10.1016/j.jtho.2020.11.026

53. Rizvi NA, Antonia SJ, Shepherd FA, Chow LQ, Goldman J, Shen Y, et al. Nivolumab (Anti-Pd-1; BMS-936558, Ono-4538) Maintenance as Monotherapy or in Combination With Bevacizumab (BEV) for Non-Small Cell Lung Cancer (Nsclc) Previously Treated With Chemotherapy. Int J Radiat OncologyBiologyPhysics (2014) 90(5):S32. doi: 10.1016/j.ijrobp.2014.08.206

54. Socinski MA, Jotte RM, Cappuzzo F, Orlandi F, Stroyakovskiy D, Nogami N, et al. Atezolizumab for First-Line Treatment of Metastatic Nonsquamous Nsclc. NEngl J Med (2018) 378(24):2288-301. doi: 10.1056/NEJMoa1716948

55. Reck M, Mok TSK, Nishio M, Jotte RM, Cappuzzo F, Orlandi F, et al. Atezolizumab Plus Bevacizumab and Chemotherapy in non-Small-Cell Lung Cancer (Impower150): Key Subgroup Analyses of Patients With EGFR Mutations or Baseline Liver Metastases in a Randomised, Open-Label Phase 3 Trial. Lancet Respir Med (2019) 7(5):387-401. doi: 10.1016/S2213-2600(19) 30084-0

56. Gadgeel SM, Stevenson JP, Langer CJ, Gandhi L, Borghaei H, Patnaik A, et al. Pembrolizumab and Platinum-Based Chemotherapy as First-Line Therapy for Advanced non-Small-Cell Lung Cancer: Phase 1 Cohorts From the KEYNOTE-021 Study. Lung Cancer (Amsterdam Netherlands) (2018) 125:273-81. doi: 10.1016/j.lungcan.2018.08.019

57. Qiu L, Zhao X, Shi W, Sun S, Zhang G, Sun Q, et al. Real-World Treatment Efficacy of Anti-Programmed Death-1 Combined With Anti-Angiogenesis Therapy in non-Small Cell Lung Cancer Patients. Medicine (2020) 99(24): e20545. doi: 10.1097/MD.0000000000020545

58. Popat S, Grohe C, Corral J, Reck M, Novello S, Gottfried M, et al. AntiAngiogenic Agents in the Age of Resistance to Immune Checkpoint Inhibitors: Do They Have a Role in non-Oncogene-Addicted non-Small Cell Lung Cancer? Lung Cancer (Amsterdam Netherlands) (2020) 144:7684. doi: 10.1016/j.lungcan.2020.04.009

59. Hegde PS, Jubb AM, Chen D, Li NF, Meng YG, Bernaards C, et al. Predictive Impact of Circulating Vascular Endothelial Growth Factor in Four Phase III Trials Evaluating Bevacizumab. Clin Cancer Res (2013) 19(4):929-37. doi: 10.1158/1078-0432.CCR-12-2535

60. Giuliano S, Pages G. Mechanisms of Resistance to Anti-Angiogenesis Therapies. Biochimie (2013) 95(6):1110-9. doi: 10.1016/j.biochi.2013.03.002

61. Liu X, Qin S, Wang Z, Xu J, Xiong J, Bai Y, et al. Early Presence of AntiAngiogenesis-Related Adverse Events as a Potential Biomarker of Antitumor Efficacy in Metastatic Gastric Cancer Patients Treated With Apatinib: A Cohort Study. J Hematol Oncol (2017) 10(1):153. doi: 10.1186/ s13045-017-0521-0

62. Mok TSK, Wu YL, Kudaba I, Kowalski DM, Cho BC, Turna HZ, et al. Pembrolizumab Versus Chemotherapy for Previously Untreated, PD-L1expressing, Locally Advanced or Metastatic non-Small-Cell Lung Cancer (KEYNOTE-042): A Randomised, Open-Label, Controlled, Phase 3 Trial. Lancet (Lond Engl) (2019) 393(10183):1819-30. doi: 10.1016/S0140-6736(18) 32409-7
63. Rossi S, Finocchiaro G, Marchetti S, Toschi L, Santoro A. Checkpoint Inhibitors: 'Raising the Bar' Also in Brain Metastases From non-Small-Cell Lung Cancer? Immunotherapy (2018) 10(5):403-10. doi: 10.2217/imt-20170151

64. Vilarino N, Bruna J, Bosch-Barrera J, Valiente M, Nadal E. Immunotherapy in NSCLC Patients With Brain Metastases. Understanding Brain Tumor Microenvironment and Dissecting Outcomes From Immune Checkpoint Blockade in the Clinic. Cancer Treat Rev (2020) 89:102067. doi: 10.1016/ j.ctrv.2020.102067

65. Marabelle A, Fakih M, Lopez J, Shah M, Shapira-Frommer R, Nakagawa K, et al. Association of Tumour Mutational Burden With Outcomes in Patients With Advanced Solid Tumours Treated With Pembrolizumab: Prospective Biomarker Analysis of the Multicohort, Open-Label, Phase 2 KEYNOTE-158 Study. Lancet Oncol (2020) 21(10):1353-65. doi: 10.1016/S1470-2045(20)30445-9

66. Asaoka Y, Ijichi H, Koike K. Pd-1 Blockade in Tumors With Mismatch-Repair Deficiency. N Engl J Med (2015) 373(20):1979. doi: 10.1056/NEJMc1510353

67. Han B, Chu T, Zhong R, Zhong H, Zhang B, Zhang W, et al. Efficacy and Safety of Sintilimab With Anlotinib as First-Line Therapy for Advanced nonSmall Cell Lung Cancer (Nsclc). J Thoracic Oncol (2019) 14(10):S129. doi: 10.1016/j.jtho.2019.08.269

68. Tsakonas G, Lewensohn R, Botling J, Ortiz-Villalon C, Micke P, Friesland S, et al. An Immune Gene Expression Signature Distinguishes Central Nervous System Metastases From Primary Tumours in non-Small-Cell Lung Cancer. Eur J Cancer (Oxford Engl 1990) (2020) 132:24-34. doi: 10.1016/j.ejca.2020.03.014

69. Rittmeyer A, Barlesi F, Waterkamp D, Park K, Ciardiello F, von Pawel J, et al. Atezolizumab Versus Docetaxel in Patients With Previously Treated nonSmall-Cell Lung Cancer (OAK): A Phase 3, Open-Label, Multicentre Randomised Controlled Trial. Lancet (London England) (2017) 389 (10066):255-65. doi: 10.1016/S0140-6736(16)32517-X

70. Gandhi L, Rodriguez-Abreu D, Gadgeel S, Esteban E, Felip E, De Angelis F, et al. Pembrolizumab Plus Chemotherapy in Metastatic non-Small-Cell Lung Cancer. N Engl J Med (2018) 378(22):2078-92. doi: 10.1056/NEJMoa1801005

71. Goldman JW, Crino L, Vokes EE, Holgado E, Reckamp K, Pluzanski A, et al Nivolumab (Nivo) in Patients (Pts) With Advanced (Adv) NSCLC and Central Nervous System (Cns) Metastases (Mets). J Thoracic Oncol (2016) 11(10):S238-S9. doi: 10.1016/j.jtho.2016.08.107

72. Hegde PS, Chen DS. Top 10 Challenges in Cancer Immunotherapy. Immunity (2020) 52(1):17-35. doi: 10.1016/j.immuni.2019.12.011

73. Fuca G, Galli G, Poggi M, Lo Russo G, Proto C, Imbimbo M, et al. Modulation of Peripheral Blood Immune Cells by Early Use of Steroids and its Association With Clinical Outcomes in Patients With Metastatic non-Small Cell Lung Cancer Treated With Immune Checkpoint Inhibitors. ESMO Open (2019) 4 (1):e000457. doi: 10.1136/esmoopen-2018-000457

74. Zhuang H, Wang Y, Cheng C, Shi S. The Efficacy of Anlotinib Instead of Glucocorticoids for Edema Induced by Brain Metastases in NSCLC Patients With anti-PD1/PDL-1 Immunotherapy. Neuro-oncology (2021) 23(1):169-71. doi: 10.1093/neuonc/noaa236

75. Billan S, Kaidar-Person O, Gil Z. Treatment After Progression in the Era of Immunotherapy. Lancet Oncol (2020) 21(10):e463-e76. doi: 10.1016/S14702045(20)30328-4

76. Venniyoor A. Synergism Between Anti-Angiogenic and Immune Checkpoint Inhibitor Drugs: A Hypothesis. Med Hypotheses (2021) 146:110399. doi: 10.1016/j.mehy.2020.110399

77. Yang Y, Li L, Jiang Z, Wang B, Pan Z. Anlotinib Optimizes Anti-Tumor Innate Immunity to Potentiate the Therapeutic Effect of PD-1 Blockade in Lung Cancer. Cancer Immunol Immunother CII (2020) 69(12):2523-32. doi: 10.1007/s00262-020-02641-5

Conflict of Interest: The authors declare that the research was conducted in the absence of any commercial or financial relationships that could be construed as a potential conflict of interest.

Copyright (c) 2021 Fang, Zhao, Ye and Chen. This is an open-access article distributed under the terms of the Creative Commons Attribution License (CC BY). The use, distribution or reproduction in other forums is permitted, provided the original author(s) and the copyright owner(s) are credited and that the original publication in this journal is cited, in accordance with accepted academic practice. No use, distribution or reproduction is permitted which does not comply with these terms. 